\title{
Prophylactic external beam radiation therapy for keloid prevention in thyroid surgery patients
}

\author{
Deena Hadedeya ${ }^{1}$, Mahmoud Shalaby $^{1}$, Mounika Akkera ${ }^{1}$, Grace Lee ${ }^{1}$, Kendra Harris ${ }^{2}$, \\ Roostam Kholmatov ${ }^{1}$, Muhammad Anwar ${ }^{1}$, Fadi Murad $^{1}$, Saad Alawaad ${ }^{1}$, Emad Kandil ${ }^{1}$ \\ ${ }^{1}$ Department of Surgery, Tulane University School of Medicine, New Orleans, LA, USA; ${ }^{2}$ Department of Radiation Oncology, Tulane University \\ School of Medicine, New Orleans, LA, USA \\ Contributions: (I) Conception and design: E Kandil, G Lee; (II) Administrative support: R Kholmatov, Muhammad Anwar, F Murad; (III) Provision \\ of study materials or patients: R Kholmatom, M Anwar, F Murad, D Hadedeya; (IV) Collection and assembly of data: R Kholmatom, M Anwar, D \\ Hadedeya; (V) Data analysis and interpretation: D Hadedeya; (VI) Manuscript writing: All authors; (VII) Final approval of manuscript: All authors. \\ Correspondence to: Emad Kandil, MD, MBA. Department of Surgery, Tulane School of Medicine, 1430 Tulane Ave, SL-22, New Orleans, LA 70112, \\ USA. Email: ekandil@tulane.edu.
}

Background: Poor cosmesis, secondary to keloid or hypertrophic scar, following thyroid surgery may cause considerable patient distress and be a significant challenge to treat. In this case series we examined the efficacy of prophylactic external beam radiation therapy (EBRT) for prevention of keloid formation in keloid-prone patients undergoing thyroid surgery. While much has been published about documenting the efficacy in reducing keloid formation following keloid excision, very little literature exists documenting prophylactic use related to surgeries with the goal of prevent de novo keloid formation.

Methods: We retrospectively evaluated a series of ten patients, who underwent a prophylactic EBRT for keloid prevention after thyroid surgery between January 2013 and February 2019. Patient demographics, primary diagnosis, surgical procedure, EBRT dosage, and post-operative visit records were reviewed.

Results: All ten patients who received EBRT for keloid prophylaxis following a thyroid surgery were female. Half of the patients were African Americans, 40\% Caucasians, and 10\% Hispanic. The mean age was $46.40 \pm 15.63$ years with BMI of $31.5 \pm 5.5 \mathrm{~kg} / \mathrm{m}^{2}$. Radiation was initiated within 6 hours of the surgery with an average radiation dose per session of $5.7 \pm 1.7$ Gy. The total average EBRT dose delivered was 17.4 4.2 Gy. Mean follow-up period was 13 months post-thyroidectomy, with the longest follow-up at 23 months. One patient, who underwent a lateral neck dissection in addition to thyroid surgery, developed hypertrophic scar in less than $10 \%$ of her incision length. Nine other patients (90\%) showed no post-surgical keloid nor hypertrophic scar formation and patients were satisfied with postsurgical cosmesis.

Conclusions: We examined the efficacy of prophylactic EBRT in keloid-prone patients undergoing thyroid surgery. Prophylactic EBRT following thyroid surgery is effective in achieving a satisfactory cosmetic outcome in patients at high risk for keloid formation.

Keywords: Thyroid surgery; neck surgery; keloid; radiation therapy; external beam radiation

Submitted May 19, 2020. Accepted for publication Sep 18, 2020.

doi: $10.21037 / g s-20-511$

View this article at: http://dx.doi.org/10.21037/gs-20-511

\section{Introduction}

Keloid is defined as benign abnormal fibrous outgrowth that extends beyond the confines of the original wound but is limited to the skin (1-3). Isolated fibroblast cells from keloid scars show increased collagen production and decreased collagenolytic activity (4). This imbalance results in an accumulation of extracellular matrix (ECM) in the scar leading to keloid formation (5). In the early phase of wound healing, the degranulating platelets produce potent 
cytokines, such as epidermal growth factor (EGF), insulinlike growth factor (IGF-I), platelet-derived growth factor (PDGF) and transforming growth factor $\beta$ (TGF- $\beta$ ), that work as chemoattractant for lymphocytes, fibroblasts, monocytes and neutrophils, which are important mediators of the normal wound healing process (6). TGF- $\beta 1$ and $\beta 2$ are crucial for collagen and proteoglycan synthesis and also play a role in preventing collagen breakdown. TGF- $\beta$ is suspected to be linked with keloid formation either by persistent production or overexpression of both TGF- $\beta 1$ and $\beta 2$ (5). Increased expression of several IGF-binding, IGF-binding-related proteins, and decreased expression of multiple IL-1-inducible genes have a role in the keloid formation as well (5). On histopathologic exam, thick collagen fibers with fibrous bands are arranged in sheets forming the upper part of dermis, mimicking fascia (7).

The overall incidence of keloid reported in literature range from 1-16\% (1,5). Different risk factors such as skin color, age, and gender have been linked to increased risk of keloid formation $(1,5)$. Keloid formation prevalence is fifteen times higher in darker-pigmented individuals. The incidence of keloid formation in African-American is as high as $5.8 \%(8,9)$. The highest rate of keloid formation is seen between 10-30 years of age (1). Women are more prone to developing keloids, which may be attributed to differences in hormone levels and a higher tendency toward having body piercings $(8,9)$. Regional susceptibility to keloid formation is also recognized in certain body parts, with the parasternal area, upper back, shoulders, earlobes and neck being the most common sites for keloid formation (10). Genetic predisposition may also play a role. An autosomal dominant pattern of inheritance has been supported by studies done in twins and families $(1,5,6)$. Keloids are also associated with genetic syndromes such as Rubinstein Taybi and Goeminne, and immune reaction may be implicated in keloid formation (11).

In addition to symptoms of pain, pruritus, and burning sensation, cosmesis is of major concern, as keloids can cause disfigurement that may impair the quality of life of the patient $(12,13)$. Treatment options include a surgical excision alone or excision with various adjuvant treatments, such as intra or postoperative steroid injection, postoperative radiation therapy, topical silicone application, intralesional cryotherapy, phototherapy, and pressure therapy (14-16). Chemotherapeutic agents, bleomycin or 5 -fluorouracil, immunomodulator imiquimod, interferons, and calcium-channel blocker, verapamil, have also been used to reduce the likelihood of keloid formation (14-16).
Multiple studies have examined the best practice evidence for reducing keloid formation in patients who underwent a surgical excision of keloids (14-17). No single best choice can be offer to the patient as each treatment modality has its own indications, side-effects and limitations (13-17).

As studies have shown keloid recurrence rate at previously excised site may be as high as $50 \%$, consideration of adjuvant therapy to further reduce keloid formation in patients with history of keloid is crucial $(18,19)$. Among adjuvant treatments available to reduce the likelihood of keloid recurrence, several studies suggest adjuvant external beam radiotherapy (EBRT) immediately after keloid excision is superior when compared to other adjuvants $(13,16,18)$. Significantly less data exists on the efficacy of adjuvant EBRT as a form of primary prevention among patients with known history of keloid formation, who are receiving a surgical incision in a notably visible area, such as the neck $(13,16,18,19)$. Studies on primary prevention of keloid formation has largely focused on the risks associated with adjuvant radiotherapy, such as sporadic risk for radiationinduced cancers $(18,19)$. The optimal timing, dosing and fractionation of radiotherapy post-operatively have not been well established (19). We assessed the role of EBRT as a prophylactic therapy for primary prevention of keloid formation following thyroid surgery. We present the following article in accordance with the STROBE reporting checklist (available at http://dx.doi.org/10.21037/gs-20-511).

\section{Methods}

\section{Demographics}

All patients underwent thyroid surgeries at a North American institution between January 2013 and February 2019. The study was conducted in accordance with the Declaration of Helsinki (as revised in 2013). The study was approved by institutional research board. Registration number 2019-2134 and individual consent for this retrospective analysis was waived. Patients who received postoperative prophylactic radiation therapy for keloid prevention following thyroid surgery were included in this study $(\mathrm{N}=10)$. Data were collected retrospectively from the institute's medical records. All female patients with a high risk of keloid formation were offered postoperative adjuvant EBRT. A High-risk patient is defined as a patient who had previous surgery or trauma and developed keloid afterward anywhere on the body. Any female patient who may become pregnant, patients who previously received irradiation to 


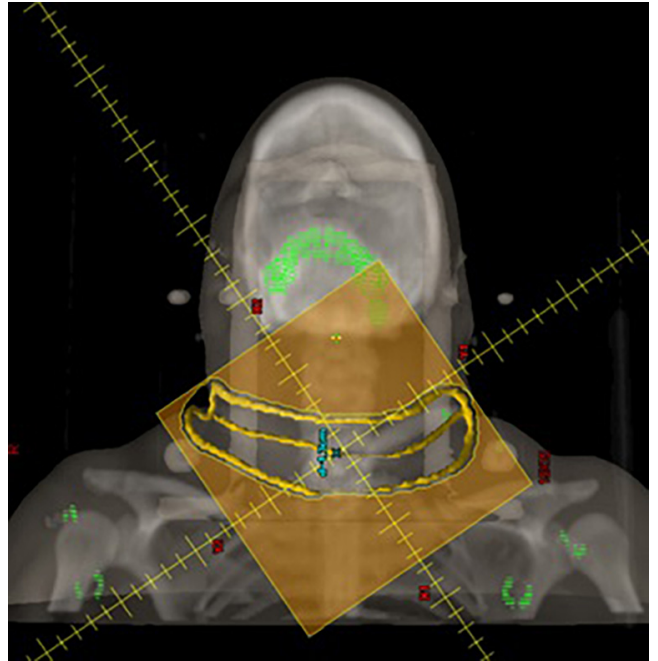

Figure 1 Digital reconstructed radiograph (DRR) showing the wired incision scar and drain site as well as the edge of the electron beam (orange), 2 centimeters out.

head and neck, and patients with no postoperative followup at 6 months were excluded from the study. An informed consent was obtained by the surgeon and the radiation oncologist prior to the therapy.

\section{EBRT}

Radiation treatments were electron beam therapy. Clinical radiotherapy mapping sessions were performed on the day of surgery. Post-operatively, wires were placed on the surgical incisions and drain site. Treatment fields were delineated by the radiation oncologist with the block edge set $2 \mathrm{~cm}$ from the scar and drain site, unless the drain site was distant $(>5 \mathrm{~cm})$ to the primary incisional scar (Figure 1). Typically, $5 \mathrm{~mm}$ bolus and $6 \mathrm{MeV}$ electron energies were favored, and the electron beam was set orthogonal to the skin. A computed tomography (CT) scan in the treatment position was performed with bolus in place, in order for an Eclipse treatment planning station to model dose delivery and aid the confirmation of the appropriate electron energy. Cerrobend electron blocks were created from this target information and additional sensitive non-target areas were shielded, if needed, with 2 -mm plastic-coated lead. The cerrobend block was inserted into the cone apparatus and treatments were typically treated at $105 \mathrm{~cm}$ source to skin distance (SSD). Prior to treatment, a cutout factor was measured and factored into the determination of appropriate monitor units (MU). Three treatment sessions

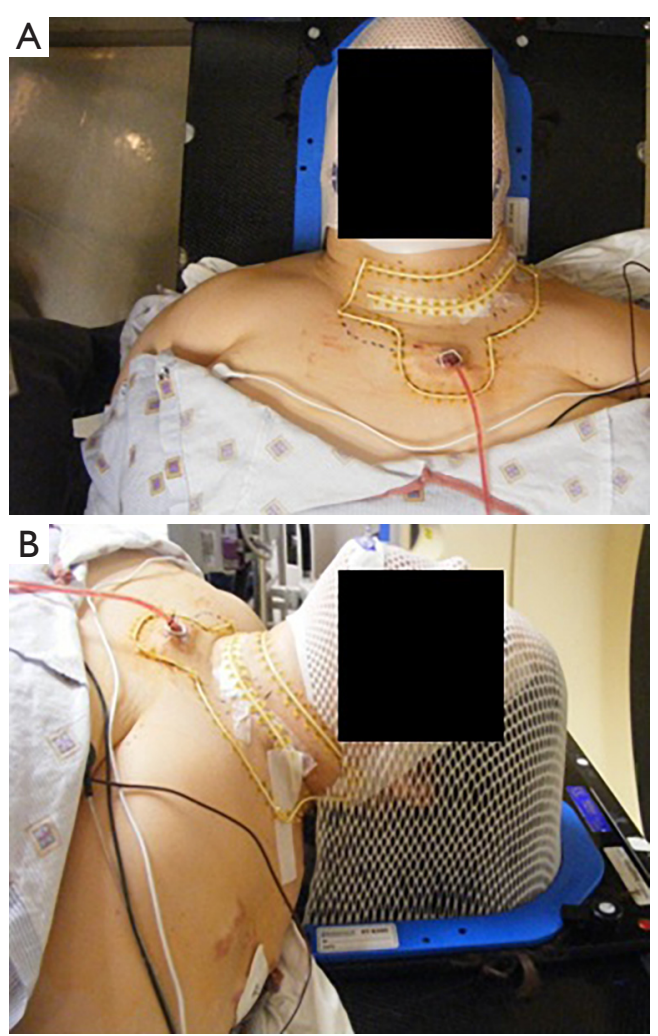

Figure 2 Anterior (A) and lateral (B) view of EBRT field.

were delivered on three sequential days (Figure 2) to a total dose of $12 \mathrm{~Gy}$.

\section{Follow-up}

Patients were evaluated for keloid formation, EBRTassociated symptoms, and satisfaction with postsurgical cosmesis postoperatively. Overgrowth of incision were considered as keloid formation. Patient demographics, diagnosis, type of surgery, dose and fractionation of radiation therapy and follow-up.

\section{Statistical analysis}

Descriptive statistics was done using SPSS version 16.0 to present our data.

\section{Results}

During the study period, ten patients with history of keloids underwent prophylactic EBRT after thyroid surgeries to prevent keloid formation. All patients studied 
were female with an average age of $46.40 \pm 15.63$ years and BMI of $31.5 \pm 5.5 \mathrm{~kg} / \mathrm{m}^{2}$. Five of the patients were African Americans, four Caucasians, and one Hispanic. Eight patients diagnosed with benign thyroid lesions (Hashimoto's thyroiditis, Graves' disease, or enlarged symptomatic nodules) underwent thyroid lobectomy or total thyroidectomy, one patient underwent total thyroidectomy with central \& lateral neck dissection, and one patient underwent a redo parathyroidectomy (Table 1).

All neck incisions were closed with 4-0 Monocryl suture in subcuticular fashion. Patients received no other adjuvant treatment for their incision, such as silicone gel sheeting, scar cream, pressure clothing, or steroids. Patients had a radiation mapping session and initial treatment on POD 0 within 6 hours of surgery. The average radiation dose per session was $5.7 \pm 1.7$ Gy. The average amount of total radiation was 17.4 \pm 4.2 Gy over three fraction sessions.

All patients underwent a surgery via anterior cervical neck incision and received three postoperative EBRT fraction sessions. Three patients underwent two surgeries, one of them had her surgeries 4 years apart and received EBRT after both procedures. EBRT was well tolerated by all patients initially, but one patient noted to have transient erythema and one had mild hyperpigmentation 2-3 weeks after the treatment, which is not uncommon. These findings were mild and temporary as they were not evident at $>6$ months follow up visits. No severe adverse effects, such as delayed wound healing, ulceration, skin peeling, wound dehiscence, or wound infection, were reported. No overt case of secondary malignancy was observed during the study period.

One patient who underwent concomitant lateral neck dissection in addition to thyroid surgery did develop hypertrophic scar which was noted in less than $10 \%$ of her index surgical incision, which measured approximately $19 \mathrm{~cm}$. The other nine patients exhibited no post-surgical keloid nor hypertrophic scar formation and patients were satisfied with postsurgical cosmesis. Average follow-up period was $12.9 \pm 7$ months.

\section{Discussion}

Keloid is an exaggerated skin healing response to surgery or any other form of trauma to skin integrity. For patients with existing keloid who pursue corrective surgical excision, an addition of adjuvant therapies following keloid excision is highly recommended, as surgical excision alone has recurrence rate of $45 \%$ to $100 \%$ (20). In 2014, the International Advisory Panel on Scar Management recommended more proactive and prophylactic options of keloid prevention such as careful attention to surgical techniques, best practices for wound care, sun protection, usage of Silicone gel or sheeting, and corticosteroid injection in high risk patients (17). Silicone gel and sheeting require 12-24 hours per day use for a minimum of 2 months, which is not ideal for a visible treatment area and non-compliant patient $(21,22)$. Similar compliance issue is noted in association with pressure therapy, as the pressure device must be worn at least 23 hours a day for 6-24 months for optimal results (6). The therapy success rate reaches $90-100 \%$, especially among burn patients (23). Another modality is Corticosteroid injections which typically requires more than one session to achieve optimized outcomes and are associated with a high frequency of adverse effects such as pain, skin atrophy, dyspigmentation, telangiectasia, delayed and poor wound healing (20,23-27). Five-year keloid recurrence rate following adjuvant corticosteroid injections is $8 \%$ to $50 \%(20,23)$. Limited data exist to support the efficacy of laser therapy as a treatment modality for cutaneous scarring management, with small case series reporting an efficacy of $72 \%$ with $585-\mathrm{nm}$ pulsed-dye laser therapy $(13,28)$. Several chemotherapeutic agents, Fluorouracil (5-FU) and interferon, have been used for intralesional injection $(5,23,29-31)$. 5-FU may cause wound ulceration and pain and interferon associated with significant expense $(5,29-31)$. Similarly, the risk of postoperative imiquimod $5 \%$ in flaps, grafts or wounds closed under tension has been a limitation as it can causes wound dehiscence, additionally, it is associated with skin irritation which may requires discontinue the medication for several days to a week $(23,32)$. In terms of oral agent, Tranilast [N-(3,4-dimethoxycinnamoyl) anthranilic acid], a collagen synthesis inhibitor has been long used in Korea and Japan, but has not been approved for keloid prevention indication by the FDA in the U.S $(31,33)$.

Adjuvant EBRT has played an important role in the prevention of keloid recurrence for decades (34). The mechanism by which radiotherapy prevents keloid formation is poorly understood, but it appears to be related to fibroblast inhibition and reduction in collagen synthesis $(35,36)$. Among patients who received EBRT in conjunction with keloid resection, published recurrence rates vary widely from $10-100 \%(25,37)$. Jones et al. reported no keloid recurrence in $81 \%$ of the 48 patients who underwent EBRT after surgical excision of keloid in different locations, including the neck (38). A $16 \%$ recurrence rate was reported by Pontoriero et al. from the 62 patients with 


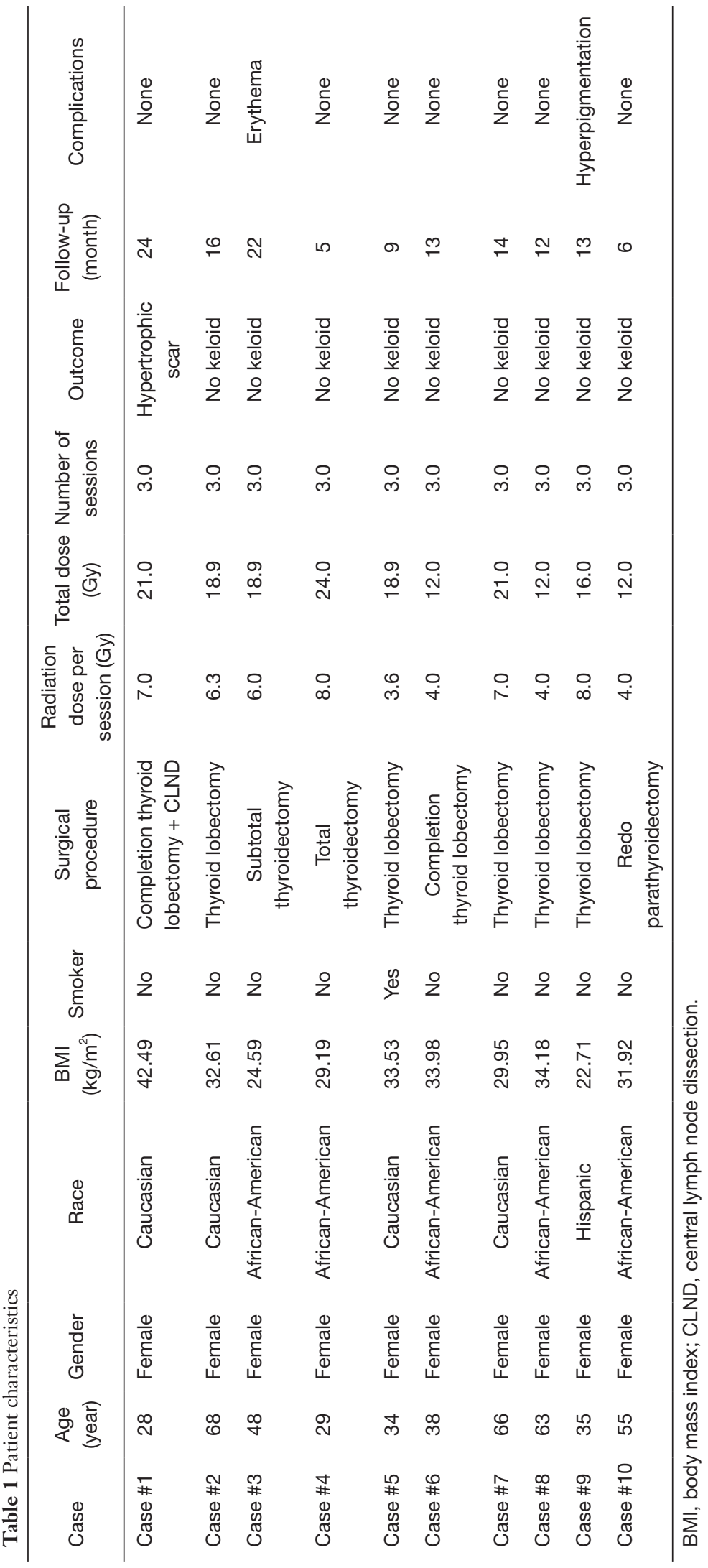


keloid formation at various sites, including face and neck (10\%), after surgical excision and EBRT (39).

Several keloid adjuvant therapy comparison studies have been performed previously. Emad et al. performed a prospective trial comparing surgical excision followed by EBRT vs. cryotherapy with intralesional triamcinolone in 2010. Patients in the adjuvant EBRT group showed higher keloid remission rate and were more satisfied with their cosmetic outcome compared to the cryotherapy and triamcinolone group (40). A randomized controlled trial by Sclafani et al. evaluated earlobe keloid excision followed by corticosteroid injection or EBRT. A lower recurrence rate was found with the EBRT group, although a statistically significant difference was not observed (41).

Less data is available on EBRT efficacy for keloid prophylaxis in patients who are undergoing surgeries in cosmetically vulnerable areas, such as the neck. The use of adjuvant EBRT in thyroid surgery patients prevented keloid in $90 \%$ of the studied patients. The only patient who experienced minimal keloid formation was undergoing a redo operation in the neck. In terms of dose and fractionation regimen, patients in this series received an average adjuvant radiation dose of 17.4 Gy divided into three fractions. Oagwa et al. and Kim et al. proposed a dose of 10-20 Gy, depending on the site $(19,42)$.

In addition to clinical effectiveness and high patient tolerance to therapy, all patients complied with the EBRT sessions. Due to a minimal number of required treatment sessions and short duration of treatments, patient compliance was high. Having limited support with transportation to the EBRT sessions may be an issue in the post-operative period.

The most commonly reported short-term side effect due to EBRT included hypopigmentation, hyperpigmentation, and erythema. We note that these were temporary and subsequently seen to have resolved at 6 months to 1 year follow-up. This finding is similar to other published studies $(16,43)$. Given the risk, exposure of normal tissues to EBRT should be carefully titrated and reduced as much as possible by using minimal dose and fractionation schedules $(42,44,45)$.

With respect to potential long-term risks, a focus on induced second malignancies and possible induction of hypothyroidism had dominated. A systematic review comprised of 33 studies, on secondary malignancy from adjuvant EBRT showed that no relationship exists between irradiation to scar and late secondary malignancy manifestation (16). Determining the risk of radiationinduced hypothyroidism is more difficult. We note that one of the largest studies looking at the relationship between hypothyroidism and direct thyroid irradiation found a strong association between thyroid dysfunction for doses above 35 Gy (as noted above, our patients received an average skin dose of $17.4 \mathrm{~Gy}$, with dose to the deeper thyroid presumably much lower given the use of superficial electrons as our prophylaxis therapy) (46). Among patients in our study, none were noted to have developed hypothyroidism in the aftermath of their radiotherapy treatments. Due to the rarity of such events, the causal relationship may not be accurately established.

There are some limitations with this study. The followup period may not be sufficient to capture possible late side effects of adjuvant EBRT. The cost of this treatment may be expensive depending on the regimen since the new radiation modalities are more expensive.

\section{Conclusions}

Keloid is a challenging problem to treat. Prevention is the key to avoiding potentially adverse effect of keloid has on patient's quality of life. Our results showed that prophylactic EBRT is highly effective in preventing keloid formation in high-risk patients, with only a minimal, short-term side effects. Potential long-term, delayed risks to normal tissue associated with EBRT and the added cost of EBRT remain minor drawbacks. Further studies are warranted on this promising modality on a wider surgical spectrum and different anatomical sites to prove it is long term efficacy on preventing keloid formation.

\section{Acknowledgments}

Funding: None.

\section{Footnote}

Reporting Checklist: We present this article in correspondence with the STROBE checklist. Available at http://dx.doi. org/10.21037/gs-20-511

Data Sharing Statement: Available at http://dx.doi. org/10.21037/gs-20-511

Conflicts of Interest: All authors have completed the ICMJE uniform disclosure form (available at http://dx.doi. org/10.21037/gs-20-511). EK serves as an Editor-in-Chief of Gland Surgery. The authors have no other conflicts of 
interest to declare.

Ethical Statement: The authors are accountable for all aspects of the work in ensuring that questions related to the accuracy or integrity of any part of the work are appropriately investigated and resolved. The study was conducted in accordance with the Declaration of Helsinki (as revised in 2013). The study was approved by Tulane university human research protection office-institutional review boards. Registration number 2019-2134 and individual consent for this retrospective analysis was waived.

Open Access Statement: This is an Open Access article distributed in accordance with the Creative Commons Attribution-NonCommercial-NoDerivs 4.0 International License (CC BY-NC-ND 4.0), which permits the noncommercial replication and distribution of the article with the strict proviso that no changes or edits are made and the original work is properly cited (including links to both the formal publication through the relevant DOI and the license). See: https://creativecommons.org/licenses/by-nc-nd/4.0/.

\section{References}

1. English RS, Shenefelt PD. Keloids and hypertrophic scars. Dermatol Surg 1999; 25:631-8.

2. Danielsen PL, Ru W, Agren MS et al. Radiotherapy and corticosteroids for preventing and treating keloid scars (Protocol). Cochrane Database Syst Rev 2013;(12):CD010883.

3. Diao JS, Xia WS, Yi CG, et al. Trichostatin A inhibits collagen synthesis and induces apoptosis in keloid fibroblasts. Arch Dermatol Res 2011;303:573-80.

4. Ladin DA, Hou Z, Patel D, et al. p53 and apoptosis alterations in keloids and keloid fibroblasts. Wound Repair Regen 1998;6:28-37.

5. Gauglitz GG, Korting HC, Pavicic T, et al. Hypertrophic scarring and keloids: pathomechanisms and current and emerging treatment strategies. Mol Med 2011;17:113-125.

6. Niessen FB, Spauwen PH, Schalkwijk J, et al. On the nature of hypertrophic scars and keloids: a review. Plast Reconstr Surg 1999;104:1435-58.

7. Huang C, Akaishi S, Hyakusoku H, et al. Are keloid and hypertrophic scar different forms of the same disorder? A fibroproliferative skin disorder hypothesis based on keloid findings. Int Wound J 2014;11:517-22.

8. Alhady SM, Sivanantharajah K. Keloids in various races. A review of 175 cases. Plast Reconstr Surg 1969;44:564-6.
9. Oluwasanmi JO. Keloid in the African. Clin Plastic Surg 1974;1:1979-95.

10. Ehrlich HP, Desmouliere A, Diegelmann RF, et al. Morphological and immunological differences between keloid and hypertrohic scar. Am J Pathol 1994;145:105-13.

11. Clark JA, Turner ML, Howard L, et al. Description of familial keloids in five pedigrees: evidence for autosomal dominant inheritance and phenotypic heterogeneity. BMC Dermatol 2009;9:8.

12. Bock O, Schmid-Ott G, Malewski P, et al. Quality of life of patients with keloid and hypertrophic scarring. Arch Dermatol Res 2006;297:433-8.

13. Leventhal D, Furr M, Reiter D. Treatment of keloids and hypertrophic scars: a meta-analysis and review of the literature. Arch Facial Plast Surg 2006;8:362-8.

14. Ogawa R. The most current algorithms for the treatment and prevention of hypertrophic scars and keloids. Plast Reconstr Surg 2010;125:557-68.

15. Nie Z, Bayat A, Behzad F, et al. Positive response of a recurrent keloid scar to topical methyl aminolevulinatephotodynamic therapy. Photodermatol Photoimmunol Photomed 2010;26:330-2.

16. van Leeuwen MC, Stokmans SC, Bulstra AE, et al. Surgical Excision with Adjuvant Irradiation for Treatment of Keloid Scars: A Systematic Review. Plast Reconstr Surg Glob Open 2015;3:e440.

17. Gold MH, McGuire M, Mustoe TA, et al. Updated international clinical recommendations on scar management: part 2--algorithms for scar prevention and treatment. Dermatol Surg 2014;40:825-31.

18. Guix B, Henríquez I, Andrés A, et al. Treatment of keloids by high-dose-rate brachytherapy: A seven-year study. Int J Radiat Oncol Biol Phys 2001;50:167-72.

19. Ogawa R, Miyashita T, Hyakusoku H, et al. Postoperative radiation protocol for keloids and hypertrophic scars: statistical analysis of 370 sites followed for over 18 months. Ann Plast Surg 2007;59:688-91.

20. Morelli Coppola M, Salzillo R, et al. Triamcinolone acetonide intralesional injection for the treatment of keloid scars: patient selection and perspectives. Clin Cosmet Investig Dermatol 2018;11:387-96.

21. Poston J. The use of silicone gel sheeting in the management of hypertrophic and keloid scars. J Wound Care 2000;9:10-6.

22. Mustoe TA. Evolution of Silicone Therapy and Mechanism of Action in Scar Management. Aesthetic Plast Surg 2008;32:82-92.

23. Jung JY, Roh MR, Kwon YS, et al. Surgery and 
perioperative intralesional corticosteroid injection for treating earlobe keloids: A Korean experience. Ann Dermatol 2009;21:221-5.

24. Mustoe TA, Cooter RD, Gold MH, et al. International clinical recommendations on scar management. Plast Reconstr Surg 2002;110:560-71.

25. Finken MJJ, Mul D. Cushing's syndrome and adrenal insufficiency after intradermal triamcinolone acetonide for keloid scars. Eur J Pediatr 2010;169:1147-9.

26. Kumar S, Singh RJ, Reed AM, Lteif AN. Cushing's syndrome after intra-articular and intradermal administration of triamcinolone acetonide in three pediatric patients. Pediatrics 2004;113:1820-4.

27. Liu MF, Yencha M. Cushing's syndrome secondary to intralesional steroid injections of multiple keloid scars. Otolaryngol Head Neck Surg 2006;135:960-1.

28. Gold MH, Berman B, Clementoni MT, et al. Updated international clinical recommendations on scar management: part 1--evaluating the evidence. Dermatol Surg 2014;40:817-24.

29. Zurada JM, Kriegel D, Davis IC. Topical treatments for hypertrophic scars. Review J Am Acad Dermatol 2006;55:1024-31.

30. Atiyeh BS. Nonsurgical Management of Hypertrophic Scars: Evidence-Based Therapies, Standard Practices, and Emerging Methods. Aesthetic Plast Surg 2007;31:468-92.

31. Saha AK, Mukhopadhyay M. A comparative clinical study on role of 5-flurouracil versus triamcinolone in the treatment of keloids. Indian J Surg 2012;74:326-9.

32. Arno AI, Gauglitz GG, Barret JP, et al. Up-to-date approach to manage keloids and hypertrophic scars: a useful guide. Burns 2014;40:1255-66.

33. Stashower ME. Successful treatment of earlobe keloids with imiquimod after tangential shave excision. Dermatol Surg 2006;32:380-6.

34. Silver Spring. FDA In Brief: FDA continues to advance oversight of drug compounding by finalizing a rule providing information on several bulk drug substances compounders not registered as outsourcing facilities can and cannot use [news release]. February 15, 2019: FDA website. Available online: pharmacytimes.com/link/256. Accessed February 15, 2019.

35. Recalcati S, Caccialanza M, Piccinno R. Postoperative radiotherapy of auricular keloids: a 26-years' experience. J Dermatolog Treat 2011;22:38-42.

36. Papadopoulou A, Kletsas D. Human lung fibroblasts prematurely senescent after exposure to ionizing radiation enhance the growth of malignant lung epithelial cells in vitro and in vivo. Int J Oncol 2011;39:989-99.

37. Sakamoto T, Oya N, Shibuya K, et al. Dose-response relationship and dose optimization in radiotherapy of postoperative keloids. Radiother Oncol 2009;91:271-6.

38. Jones ME, Ganzer CA, Bennett D, et al. Surgical Excision of Keloids Followed by In-office Superficial Radiation Therapy: Prospective Study Examining Clinical Outcomes. Plast Reconstr Surg Glob Open 2019;7:e2212.

39. Pontoriero A, Potami, A, Iatì G, et al. Post-operative radiotherapy of keloids. A 10-years experience of kilovoltage irradiation. Int J Radiat Res 2015;13:201-4.

40. Emad M, Omidvari S, Dastgheib L, et al. Surgical excision and immediate postoperative radiotherapy versus cryotherapy and intralesional steroids in the management of keloids: a prospective clinical trial. Med Princ Pract 2010;19:402-5.

41. Sclafani AP, Gordon L, Chadha M, et al. Prevention of earlobe keloid recurrence with postoperative corticosteroid injections versus radiation therapy: a randomized, prospective study and review of the literature. Dermatol Surg 1996;22:569-74.

42. Kim K, Son D, Kim J. Radiation Therapy Following Total Keloidectomy: A Retrospective Study over 11 Years. Arch Plast Surg 2015;42:588-95.

43. Song $\mathrm{C}, \mathrm{Wu} \mathrm{HG}$, Chang $\mathrm{H}$, et al. Adjuvant single-fraction radiotherapy is safe and effective for intractable keloids. J Radiat Res 2014;55:912-6.

44. Malaker A, Ellis F, Paine CH. Keloid scars: a new method of treatment combining surgery with interstitial radiotherapy. Clin Radiol 1976;27:179-83.

45. McKeown SR, Hatfield P, Prestwich RJ, et al. Radiotherapy for benign disease; assessing the risk of radiation-induced cancer following exposure to intermediate dose radiation. Br J Radiol 2015;88:20150405.

46. Inskip PD, Veiga LHS, Brenner AV, et al. Hypothyroidism after Radiation Therapy for Childhood Cancer: A Report from the Childhood Cancer Survivor Study. Radiat Res 2018;190:117-32.

Cite this article as: Hadedeya D, Shalaby M, Akkera M, Lee G, Harris K, Kholmatov R, Anwar M, Murad F, Alawaad S, Kandil E. Prophylactic external beam radiation therapy for keloid prevention in thyroid surgery patients. Gland Surg 2021;10(1):65-72. doi: 10.21037/gs-20-511 